Dirk Preußners

Mehr Erfolg im Technischen Vertrieb 
Dirk Preußners

\section{Mehr Erfolg im Technischen Vertrieb}

15 Schritte,

die Sie voranbringen 
Bibliografische Information der Deutschen Nationalbibliothek

Die Deutsche Nationalbibliothek verzeichnet diese Publikation in der

Deutschen Nationalbibliografie; detaillierte bibliografische Daten sind im Internet über <http://dnb.d-nb.de> abrufbar.

1. Auflage 2009

Alle Rechte vorbehalten

(C) Gabler I GWV Fachverlage GmbH, Wiesbaden 2009

Lektorat: Barbara Möller

Gabler ist Teil der Fachverlagsgruppe Springer Science+Business Media. www.gabler.de

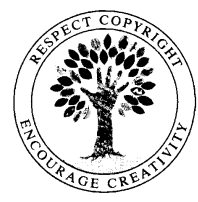

Das Werk einschließlich aller seiner Teile ist urheberrechtlich geschützt. Jede Verwertung außerhalb der engen Grenzen des Urheberrechtsgesetzes ist ohne Zustimmung des Verlags unzulässig und strafbar. Das gilt insbesondere für Vervielfältigungen, Übersetzungen, Mikroverfilmungen und die Einspeicherung und Verarbeitung in elektronischen Systemen.

Die Wiedergabe von Gebrauchsnamen, Handelsnamen, Warenbezeichnungen usw. in diesem Werk berechtigt auch ohne besondere Kennzeichnung nicht zu der Annahme, dass solche Namen im Sinne der Warenzeichen- und Markenschutz-Gesetzgebung als frei zu betrachten wären und daher von jedermann benutzt werden dürften.

Umschlaggestaltung: Nina Faber de.sign, Wiesbaden

Umschlagbild: VisionsofAmerica/Joe Sohm/Digital Vision/Gettylmages, Fotosearch

Satz: ITS Text und Satz Anne Fuchs, Bamberg

Druck und buchbinderische Verarbeitung: Krips b.v., Meppel

Gedruckt auf säurefreiem und chlorfrei gebleichtem Papier

Printed in the Netherlands

ISBN 978-3-8349-1147-6 
Für Diana 


\section{Inhaltsverzeichnis}

Vorwort oder warum Sie dieses Buch lesen sollten

1. Wie Sie Ihren Vertrieb sehen sollten, um Erfolge zu steigern

Vertrieb sagen, Bedarfsdeckung meinen

Gewinnen oder nur nicht verlieren?

Kästchendenken in der Praxis

Nur Profis sorgen für Profit

2. Wie Sie Ihren Kunden zeigen, dass Sie Experte sind

Sich als Lösungsfinder positionieren

Was bieten Sie, was Wettbewerber nicht bieten?

„Nur Innovationen bringen uns weiter. “ Interview mit

Dietrich Eberhardt, Kaeser Kompressoren GmbH,

Coburg

3. Wie Sie Ihre Vertriebsziele richtig stecken

Nicht nur der Umsatz zählt

Vertriebsziele weder über- noch unterschätzen

4. Wie Sie neue Kunden finden und qualifizieren

Werden Sie zum „Rosinen-Picker“

Wiederholungskäufe sind Gold wert

Verkauf und Zufallsverkauf unterscheiden

Von national zu international

Nicht alles ist von Erfolg gekrönt

„International helfen Agenten. "Interview mit

Claus Bornhorst, Rheinmetall Defence Electronics

GmbH, Bremen

5. Wie Sie Einwände aushebeln und Kunden zähmen

Rezepte gegen Einwände

Einwände und Ausreden unterscheiden 
6. Wie Sie Kunden per Telefon akquirieren

(oder akquirieren lassen)

Durch Fleiß das mangelnde Talent kompensieren __ 52

Ein roter Faden für die Praxis _ 53

Kunden möchten mit Entscheidern reden _ $\quad 55$

$\begin{array}{ll}\text { Professionalität zahlt sich aus _ـ } & 59\end{array}$

$\begin{array}{ll}\text { Wie Sie Ihre Abschlussrate weiterbringt _ } & 60\end{array}$

Noch besser telefonieren _ 61

7. Wie Sie Kunden auf Messen akquirieren 63

$\begin{array}{ll}\text { Fokus auf den Messebesucher legen _ـ } 63 & 63\end{array}$

Messen gehen ins Geld _ 63

Machen Sie den Stand-Test $\quad 64$

Kann ich Ihnen helfen? - Nein! _ 66

Redepause: Was Sie erzählen, wissen Sie doch bereits __ $\quad 70$

Besuchsbericht: Mehr als nur Pflicht _ 71

Und wie geht es weiter? - Ich rufe Sie an!_ 72

8. Wie Sie Ihre Bedarfsanalyse optimieren $\quad 74$

Finden Sie den roten Knopf Ihres Kunden _ 74

Die Zeiten ändern sich, der Bedarf auch _ 75

Was Sie interessiert, interessiert nicht unbedingt

den Kunden

„Mehr Innovationen im Vertrieb. “ Interview mit

Rainer Hoffmann, Carhs GmbH, Alzenau _ 81

9. Wie Sie das kundenseitige Entscheidungsgefüge knacken 84

Einen kennt man, aber viele entscheiden _ـ 84

Wer mischt nur mit und wer entscheidet?__ 86

Häuptlinge sprechen nicht mit Indianern _ 88

10. Wie Sie Anfragen selektieren, die Erfolge versprechen 90

$\begin{array}{ll}\text { Erst prüfen, dann anbieten } & 90\end{array}$

Bid oder No-Bid - ein Angebots-Report

bringt Transparenz

11. Wie Sie Ihre Angebote verbessern $\quad 96$

$\begin{array}{ll}\text { Die Basis des Angebots schaffen __ } & 96\end{array}$

\begin{tabular}{lr} 
Angebotsformen festlegen _ $\quad 98$ \\
\hline
\end{tabular}

Einzelleistungen im Angebot hervorheben _ 103

Das Anschreiben muss Lust auf das Angebot machen _ 104 
Was Ihnen eine Auftragsverlustanalyse bringt

Wie Sie interne Abläufe in der Angebotsphase optimieren

„Der Kunde findet uns!“ Interview mit Thomas Johnsen, encoway GmbH, Bremen

12. Wie Sie Präsentationen ,entkrampfen“

PowerPoint-Karaoke kommt selten an

Reißen Sie Ihre Kunden mit

Wie Sie Ihre Fachkompetenz noch sichtbarer machen

13. Wie Sie hohe Preise durchsetzen

Schaffen Sie eine Erfolgsbasis

Kunden kaufen Nutzen und keine Produkte

Klassiker Nr. 1: Am Preis müssen Sie noch was machen

Jeder Nachlass wirkt sich fatal auf Ihren Gewinn aus

Was neben dem Preis noch zählt

Preis drücken: So reagieren Sie richtig

Hohe Preise müssen Sie begründen

Legen Sie immer den nächsten Schritt fest

„Demag-Mitarbeiter sind TÜV-zertifiziert. “ Interview

mit Peter Hering, Demag Cranes \& Components

GmbH, Wetter/Ruhr

14. Wie Sie Ihre bestehenden Kunden binden

Vom Newsletter zum News-Flash

Informationsgehalt rauf, Werbung runter

Kundenevents gezielt einsetzen

15. Wie Sie Ihre Vertriebskompetenz erweitern

Kontinuierlich besser werden

Die richtigen Maßnahmen treffen

Den optimalen Experten finden

Schlusswort

Verbesserungen umsetzen, jetzt!

Dank

Literatur 


\section{Vorwort oder warum Sie dieses Buch lesen sollten}

Glück ist ein flüchtiger Bekannter. Erfolg aber basiert auf Engagement, Leidenschaft und Hartnäckigkeit - gerade im Technischen Vertrieb. Im beruflichen Alltag jedoch fällt Vieles der Routine zum Opfer, wird der Blick für das Wesentliche gern verstellt. Dieses Buch verfolgt den Ansatz, die erfolgreichen Säulen des Vertriebs praxisnah aufzuschlüsseln, Verbesserungspotenzial auf vielen Ebenen aufzuzeigen und neu für sie zu sensibilisieren. Manchmal muss man neue Wege gehen, um erfolgreicher zu sein, manchmal alte Wege ausbauen. In jedem Fall aber muss man offen sein und neugierig bleiben. Denn wer stehen bleibt, kann nur verlieren.

Technische Produkte und Dienstleistungen zu vertreiben, stellt besondere Herausforderungen an alle beteiligten Personen. Vorstände, Geschäftsführer, Vertriebsleiter und Vertriebsmitarbeiter sind kontinuierlich gefordert, ihre Produkte und Dienstleistungen den nationalen und internationalen Märkten anzupassen, neue Kunden $\mathrm{zu}$ akquirieren, bestehende Kunden $\mathrm{zu}$ binden, Alleinstellungsmerkmale herauszustellen und den Kundennutzen aufzuzeigen, die Umsätze zu steigern, Ausgaben zu senken und Gewinne zu optimieren.

Das Buch richtet sich an alle Fach- und Führungskräfte im Technischen Vertrieb, die ihre Vertriebserfolge steigern möchten. Und insbesondere an alle Personen, die im Vertrieb erklärungsbedürftiger technischer Produkte täglich ihren Mann oder ihre Frau stehen, ihr Vorgehen und ihre bereits vorhandenen Ideen reflektieren und neue Denkanstöße bekommen möchten. Es ist in der Sprache der technischen Fach- und Führungskräfte geschrieben und hat nicht den Anspruch, auf andere Branchen übertragbar zu sein. Denn jede Branche hat ihre eigenen Spielregeln.

Dieses Buch sollten Sie lesen, weil es präzise und praxisnah auf ausgewählte Bereiche des Technischen Vertriebs mit hohem Verbesserungspotenzial eingeht und Ihnen die Möglichkeit gibt, Mankos in Ihrem Vertrieb aufzudecken und Verbesserungen zeitnah umzusetzen. Es stellt jedoch keine komplette Abhandlung des Themas „Technischer Vertrieb“ dar. 
Die Idee, dieses Buch zu verfassen, kam mir bereits vor Jahren, als ich als Vertriebsingenieur für technische Investitionsgüter erfolgreich tätig war. Gefestigt wurde sie, während ich als Vertriebsleiter nicht nur für die Führung von Vertriebsmitarbeitern verantwortlich war, sondern auch den Markt in Asien für ein deutsches Industrieunternehmen aufbaute. Jetzt, als selbstständiger Experte für Technischen Vertrieb, kann ich zu den jahrelangen Erfahrungen in internationalen Märkten meine Erkenntnisse einfließen lassen, die ich in den letzten Jahren durch vertriebsbezogenes Expertencoaching und Seminare in zahlreichen Unternehmen sammeln durfte.

Wenn Sie als Fach- und Führungskraft im Technischen Vertrieb tätig sind, dann bin ich einer von Ihnen. Mit dem nötigen Abstand zu Ihrem Tagesgeschäft bin ich derjenige, der auf den sprichwörtlichen „Leuchtturm“ geklettert ist, um sich den Technischen Vertrieb aus der Vogelperspektive anzusehen. Eine Plattform, die es mir erlaubt, Ihnen deutliche Erfolgspotenziale aufzuzeigen.

Sicherlich gibt es zum Thema „Vertrieb“ bereits zahlreiche Bücher. Doch meine Erfahrung ist, dass der Technische Vertrieb besondere Herausforderungen stellt. Diese liegen:

- im hohen technischen Anpassungs-/Entwicklungsanteil der Produkte (im Gegensatz zu standardisierten Produkten),

- in den ausgeprägten Integrationsanforderungen,

$\checkmark$ in der starken Ausrichtung auf Projekte (wenige Projekte mit hohen Umsätzen),

- im hohen Exportanteil und damit verbunden in der starken Internationalität,

- in den kleinen gebiets-, produkt- oder branchenspezifischen Vertriebsteams,

- in den komplexen Angeboten, die nach vom Kunden vorgegebenen technischen Spezifikationen erarbeitet werden.

All dies weist darauf hin, welche Besonderheiten wir - abweichend von anderen Branchen - im Technischen Vertrieb berücksichtigen müssen, um langfristig erfolgreich zu sein. 
Welchen Nutzen bringt Ihnen dieses Buch? Das Buch hilft Ihnen:

- Ihre Vertriebseffizienz zu erhöhen.

- Ihre Kunden optimal zu selektieren.

- Ihre Kunden verstärkt zu analysieren.

- Ihre Produkte aus der Sicht des Kunden darzustellen.

- Ihre Alleinstellungsmerkmale in den Vordergrund zu stellen.

- Ihre Kunden in Besprechungen, Präsentationen und Verhandlungen zu überzeugen.

Kurzum: Das Buch hilft Ihnen, Ihre Vertriebskosten zu senken und Ihre Umsätze und Gewinne zu steigern.

Mehr Vertriebserfolg ergibt sich aus der Summe zahlloser kleiner Optimierungsschritte und ist nicht an die eine große Verbesserung geknüpft - wie oft fälschlicherweise angenommen wird. So bietet Ihnen jedes Kapitel eine Chance, Ihren Vertrieb zu verbessern und Ihre Vertriebserfolge zu steigern. Was auch bedeutet, dass Sie dieses Buch nicht in seiner gewählten Chronologie lesen müssen. Interessiert Sie das Thema Weiterbildung, beginnen Sie mit dem Schlusskapitel. Wollen Sie Ihre Angebotserstellung verbessern, gehen Sie direkt zu Kapitel 11. Beginnen Sie dort, wo Sie Verbesserungspotenzial für sich sehen, und gestalten Sie sich Ihr persönliches Inhaltsverzeichnis. Schließlich geben auch die Interviews mit erfolgreichen Führungskräften aus der Praxis jede Menge Erfahrung weiter.

Am Ende eines jeden Kapitels konfrontiere ich Sie mit dem Punkt „Verbesserungen umsetzen“. Darin stelle ich Ihnen gezielt Fragen, deren Beantwortung Verbesserungen für die Praxis initiieren.

Wenn in diesem Buch nur ein Denkanstoß für Sie dabei ist, so hat sich seine Lektüre bereits für Sie gelohnt. Starten Sie erfolgreich durch!

Zahlreiche neue Ansätze, um Ihre Vertriebserfolge zu steigern, wünscht Ihnen

Ihr Dirk Preußners

München, im März 2009 
Anmerkung: Um Ihnen das Lesen zu erleichtern, habe ich meistens ein einheitliches grammatikalisches Geschlecht gewählt.

Wenn im Text von „Produkt" die Rede ist, so ist damit ebenfalls Dienstleistung, Anlage, System etc. gemeint. Mit dem Begriff „Kunde“ ist auch ein Interessent gemeint, der noch keinen Kauf vollzogen hat.

Für Anregungen und Verbesserungsvorschläge zu diesem Buch sowie Erfahrungsberichte aus Ihrer Praxis bin ich Ihnen dankbar. Schreiben Sie mir bitte eine E-Mail an dp@preussners.de.

14 Vorwort oder warum Sie dieses Buch lesen sollten 